\title{
Corrigendum and Addendum
}

Correction to the paper Continuous dependence of the local strong unicity constant on domain (Aequationes Math. 46 (1993), 229-242)

\section{Chang Zhong Zhu And Charles B. Dunham}

Recently, we found a correctable error in the proof of Theorem 4 (which is a key theorem) of our paper [1]: on p. 239, last 7-8 lines, it said

"Thus, by Theorem 2, we have

$$
\lim _{k \rightarrow \infty} \gamma\left(f-F\left(A_{k}, \cdot\right), S\left(A_{k}\right), X_{k}\right)=\gamma\left(f-F\left(A^{*}, \cdot\right), S\left(A^{*}\right), X\right) . "
$$

But here $f-F\left(A_{k}, \cdot\right)$ is dependent on $k$, while the $f$ in Theorem 2 (see p. 234) is fixed, so we can not get the above equality by that Theorem 2 .

In order to correct this error, we study the continuous dependence of the local strong unicity constant on approximated function and domain simultaneously. This itself is of significance in approximation theory. To do so, we keep all the notations and most descriptions in that paper unchanged, and make the following changes.

(1) We add a definition after Definition 3 (see p. 230):

Definition 4. Let $f_{k} \in C(X)(k=1,2, \ldots)$, we say $f_{k} \rightarrow f$ if $\left\|f_{k}-f\right\|_{X} \rightarrow 0$.

(2) The original Lemma 1 (see p. 230) should be changed to:

LeMma 1. Let $f_{k} \rightarrow f, M_{k} \rightarrow M$ and $X_{k} \rightarrow X$. Let $A_{k}^{*}=\left(a_{1, k}^{*}, \ldots, a_{n, k}^{*}\right)$ be a best parameter to $f_{k}$ from $M_{k}$ on $X_{k}$. Then $\left\{A_{k}^{*}\right\}$ has an accumulation point, say $A^{*}=\left(a_{1}^{*}, \ldots, a_{n}^{*}\right)$, any such accumulation point is best to from $M$ on $X$, and 


$$
\left\|f_{k}-\sum_{i=1}^{n} a_{i, k}^{*} \phi_{i, k}\right\|_{X_{k}} \rightarrow\left\|f-\sum_{i=1}^{n} a_{i}^{*} \phi_{i}\right\|_{X}
$$

This was proved in $[5$, p. $129-130]$ (where the reference [5] is in [1]).

(3) The original Theorem 1 (see p. 232) should be changed to:

THEOREM 1. Let $f_{k} \rightarrow f, M_{k} \rightarrow M$ and $X_{k} \rightarrow X$, if $\sum_{i=1}^{n} a_{i, k}^{*} \phi_{i, k}$ is the SUBA to $f_{k}$ from $M_{k}$ on $X_{k}, \gamma\left(f_{k}, M_{k}, X_{k}\right)$ is the corresponding SUC, and $\sum_{i=1}^{n} a_{i}^{*} \phi_{i}$ is the SUBA to $f$ from $M$ on $X, \gamma(f, M, X)$ is the corresponding SUC, then

$$
\limsup _{k \rightarrow \infty} \gamma\left(f_{k}, M_{k}, X_{k}\right) \leq \gamma(f, M, X)
$$

The proof is similar to the original, and we need to use the new Lemma 1.

(4) Now we change the original Theorem 2 (see p. 234). Let $f_{k} \in C(X), f_{k} \notin M_{k}$, $k=1,2, \ldots$, and $\sum_{i=1}^{n} a_{i, k} \phi_{i, k}(x)$ be the SUBA to $f_{k}$ from $M_{k}$ on $X_{k}$. Let

$$
S_{X_{k}}\left(M_{k}\right)=\left\{m \in M_{k}:\|m\|_{X_{k}}=1\right\}
$$

and

$$
E_{\boldsymbol{M}_{k}, X_{k}}\left(f_{k}\right)=\left\{x \in X_{k}:\left|f_{k}(x)-\sum_{i=1}^{n} a_{i, k}^{*} \phi_{i, k}(x)\right|=\left\|f_{k}-\sum_{i=1}^{n} a_{i, k}^{*} \phi_{i, k}\right\|_{X_{k}}\right\},
$$

and

$$
R_{k}(x)=f_{k}(x)-\sum_{i=1}^{n} a_{i, k}^{*} \phi_{i, k}(x)
$$

THEOREM 2. Under the assumptions of Theorem 1 , let $M$ and $M_{k}$ be Haar subspaces of $C(X), f \notin M$, and $d\left(E_{M_{k}, X_{k}}\left(f_{k}\right), E_{M, X}(f)\right) \rightarrow 0$ as $k \rightarrow \infty$. Then

$\lim _{k \rightarrow \infty} \gamma\left(f_{k}, M_{k}, X_{k}\right)=\gamma(f, M, X)$.

Proof. Let $L=\lim \inf _{k \rightarrow \infty} \gamma\left(f_{k}, M_{k}, X_{k}\right)$. By Theorem 1 we need only prove 
that $L \geq \gamma(f, M, X)$. Select a subsequence and relabel it so that $L=$ $\lim _{k \rightarrow \infty} \gamma\left(f_{k}, M_{k}, X_{k}\right)$. For fixed $k$, choose $m_{k} \in S_{X_{k}}\left(M_{k}\right)$ for which (here we use the formula at the top of p. 233 of [1].)

$$
\max _{x \in E_{M_{k}, X_{k}}\left(f_{k}\right)}\left(\operatorname{sgn} R_{k}(x)\right) m_{k}(x)<\gamma\left(f_{k}, M_{k}, X_{k}\right)+\frac{1}{k}
$$

If $m_{k}=\sum_{i=1}^{n} a_{i, k} \phi_{i, k}$ then, since $\left\|m_{k}\right\|_{X_{k}}=1$, Lemma 3 in [1] implies that $\left\{a_{i, k}\right\}$ is bounded for $i=1, \ldots, n$. Since $M_{k} \rightarrow M$, we can further extract a subsequence and relabel it so that $m_{k} \rightarrow m$ uniformly on $X$ for some $m \in M$. By Lemma 2 in [1], $\left\|m_{k}\right\|_{X_{k}} \rightarrow\|m\|_{X}$ and so $m \in S_{X}(M)$. Now select $y \in E_{M, X}(f)$ for which $(\operatorname{sgn} R(y)) m(y)=\max _{x \in E_{M, X}(f)}(\operatorname{sgn} R(x)) m(x)$. But $d\left(E_{M_{k}, X_{k}}\left(f_{k}\right), E_{M, X}(f)\right) \rightarrow 0$, and so we can select $x_{k} \in E_{M_{k}, X_{k}}\left(f_{k}\right)$ so that $x_{k} \rightarrow y$. Since $m_{k} \rightarrow m$ and $R_{k} \rightarrow R$ uniformly on $X$, the sequences $\left\{m_{k}\right\}$ and $\left\{R_{k}\right\}$ are equicontinuous and thus $m_{k}\left(x_{k}\right) \rightarrow m(y)$ and $R_{k}\left(x_{k}\right) \rightarrow R(y)$. Since $f \notin M, R(y) \neq 0$ and so $\operatorname{sgn} R_{k}\left(x_{k}\right)=\operatorname{sgn} R(y)$ for $k$ sufficiently large. We now have that

$$
\begin{aligned}
\gamma(f, M, X) & \leq(\operatorname{sgn} R(y)) m(y)=\lim _{k \rightarrow \infty}\left(\operatorname{sgn} R_{k}\left(x_{k}\right)\right) m_{k}\left(x_{k}\right) \\
& \leq \limsup _{k \rightarrow \infty} \max _{x \in E_{M_{k}, x_{k}}\left(f_{k}\right)}\left(\operatorname{sgn} R_{k}(x)\right) m_{k}(x) \\
& \leq \lim _{k \rightarrow \infty}\left(\gamma\left(f_{k}, M_{k}, X_{k}\right)+\frac{1}{k}\right)=L,
\end{aligned}
$$

and the proof is complete.

REMARK. The condition $f \notin M$ is necessary. Here is a counterexample. If $X=[-1,1], M=\pi_{1}$ (the set of polynomials of degree 1 or less) and $f=0$, then $f$ is its own best approximation from $M$ on $X, E_{M, X}=[-1,1]$, and $\gamma(f, M, X)=1$. Let $M_{k}=M=\pi_{1}, X_{k}=X=[-1,1]$, and $f_{k}=1 / k$ on $\left[-1,-\frac{1}{3}-(1 / k)\right] \cup\left[\frac{1}{3}+(1 / k), 1\right]$, $f_{k}=-1 / k$ on $\left[-\frac{1}{3}+(1 / k), \frac{1}{3}-(1 / k)\right]$, and $f_{k}$ be linear on $\left[-\frac{1}{3}-(1 / k),-\frac{1}{3}+(1 / k)\right]$ and on $\left[\frac{1}{3}-(1 / k), \frac{1}{3}+(1 / k)\right]$. Then 0 is the best approximation to $f_{k}$ from $M_{k}$ on $X_{k}$, and $E_{M_{k}, X_{k}}=\left[-1,-\frac{1}{3}-(1 / k)\right] \cup\left[-\frac{1}{3}+(1 / k), \frac{1}{3}-(1 / k)\right] \cup\left[\frac{1}{3}+(1 / k), 1\right]$. Then $d\left(E_{M_{k}, X_{k}}\left(f_{k}\right), E_{M, X}(f)\right) \rightarrow 0$. Using the formula near the top of p. 233 in [1] and some line sketching, we get

$$
\gamma\left(f_{k}, M_{k}, X_{k}\right)=\frac{4 k-3}{8 k+3}
$$

Thus $\lim _{k \rightarrow x} \gamma\left(f_{k}, M_{k}, X_{k}\right)=\frac{1}{2} \neq 1=\gamma(f, M, X)$. 
Also, this condition is necessary for Theorem 2 in [1]. A counterexample can be obtained with $f(x)=2 x, M=\pi_{1}, X=X_{k}=[-1,1]$, and $M_{k}=\operatorname{span}\left\{1,2 x+f_{k}(x)\right\}$.

(5) The original Theorem 3 (see p. 237) should be changed to:

Theorem 3. Let $A^{*}$ be locally best to $f$ on $X$, and $F$ satisfy the local Haar condition at $A^{*}$. Let $f_{k} \rightarrow f, X_{k} \rightarrow X$. Then for $k$ sufficiently large there is a locally best parameter $A_{k}$ to $f_{k}$ on $X_{k}$ such that $A_{k} \rightarrow A^{*}$.

Proof. Since $A^{*}$ is a strong local best parameter to $f$ (by Remark 4 in [1]), there is an $\varepsilon_{0}>0$ such that $\left\|f-F\left(A^{*}, \cdot\right)\right\|_{X}<\|f-F(B, \cdot)\|_{X}$ for all $B$ with $\left\|B-A^{*}\right\|_{c}<\varepsilon_{0}$. Fix $0<\varepsilon<\varepsilon_{0}$. We claim that for $k$ sufficiently large, $\left\|f_{k}-F\left(A^{*}, \cdot\right)\right\|_{X_{k}}<\left\|f_{k}-F(B, \cdot)\right\|_{X_{k}}$ for all $B$ with $\left\|B-A^{*}\right\|_{c}=\varepsilon$. This is sufficient since the compactness of the set $\left\{B:\left\|B-A^{*}\right\|_{c} \leq \varepsilon\right\}$ and the continuity of $F(B, \cdot)$ with respect to $B$ (we note the fact that $F(B, \cdot)$ depends continuously on $B$ in a neighbourhood of $A^{*}$ ) imply that $f_{k}$ has a best parameter in this set and the claim implies that the best parameter is interior to this set.

Suppose the claim is false. Select a subsequence and relabel, and for each $k$ select parameter $B_{k}$ for which

$$
\text { (*) }\left\|f_{k}-F\left(A^{*}, \cdot\right)\right\|_{X_{k}} \geq\left\|f_{k}-F\left(B_{k}, \cdot\right)\right\|_{X_{k}} \text {. }
$$

Extracting a subsequence and relabeling so that $B_{k} \rightarrow B$, we obtain $\left\|B-A^{*}\right\|_{c}=\varepsilon$. Passing to the limit in (*) and applying Lemma 2 in [1],

$$
\left\|f-F\left(A^{*}, \cdot\right)\right\|_{X} \geq\|f-F(B, \cdot)\|_{X}
$$

which is a contradiction, and the proof is complete.

(6) Finally, we change the original Theorem 4 (see p. 239) to:

THEOREM 4. Let $f \notin(F, P), A^{*}$ be locally best to $f$ on $X$. Let $F$ satisfy the local Haar condition on a neighbourhood $N_{A^{*}}$ of $A^{*}$ with $N_{A^{*}} \subset P$. Let $B_{k} \rightarrow A^{*}$ imply $\left\|F_{i}\left(B_{k}, \cdot\right)-F_{i}\left(A^{*}, \cdot\right)\right\|_{X} \rightarrow 0, i=1, \ldots, n$. Let $f_{k} \rightarrow f, X_{k} \rightarrow X$ and $d\left(E_{F, X_{k}}\left(f_{k}\right)\right.$, $\left.E_{F, X}(f)\right) \rightarrow 0$. Then

$$
\text { (**) } \lim _{k \rightarrow \infty} \gamma_{l}\left(f_{k}, A_{k}, F, X_{k}\right)=\gamma_{l}\left(f, A^{*}, F, X\right),
$$

where $A_{k}$ is locally best parameter to $f_{k}$ on $X_{k}$ from Theorem 3. 
Proof. By Theorem 3, $A_{k} \rightarrow A^{*}$, and for $k$ sufficiently large, $A_{k} \in N_{A^{*}}$. So, $F$ satisfies the local Haar condition at $A_{k}$. By Lemma 4 in [1],

$$
\gamma_{l}\left(f_{k}, A_{k}, F, X_{k}\right)=\gamma\left(f_{k}-F\left(A_{k}, \cdot\right), S\left(A_{k}\right), X_{k}\right)
$$

Since $A_{k} \rightarrow A^{*}$, and by Remark 3 in [1] and the assumptions of this theorem, we have

$$
\left\|\left(f_{k}-F\left(A_{k}, \cdot\right)\right)-\left(f-F\left(A^{*}, \cdot\right)\right)\right\|_{X} \rightarrow 0
$$

and

$$
\left\|F_{i}\left(A_{k}, \cdot\right)-F_{i}\left(A^{*}, \cdot\right)\right\|_{X} \rightarrow 0, \quad i=1, \ldots, n,
$$

i.e. $S\left(A_{k}\right) \rightarrow S\left(A^{*}\right)$. Besides, since $d\left(E_{F, X_{k}}\left(f_{k}\right), E_{F, X}(f)\right) \rightarrow 0$, by Remark 5 in [1], we have

$$
d\left(E_{S\left(A_{k}\right), X_{k}}\left(f_{k}-F\left(A_{k}, \cdot\right)\right), E_{S\left(A^{*}\right), X}\left(f-F\left(A^{*}, \cdot\right)\right) \rightarrow 0 .\right.
$$

Thus, by Theorem 2, we have

$$
\lim _{k \rightarrow \infty} \gamma\left(f_{k}-F\left(A_{k}, \cdot\right), S\left(A_{k}\right), X_{k}\right)=\gamma\left(f-F\left(A^{*}, \cdot\right), S\left(A^{*}\right), X\right)
$$

But, by Lemma 4 in [1],

$$
\gamma\left(f-F\left(A^{*}, \cdot\right), S\left(A^{*}\right), X\right)=\gamma_{l}\left(f, A^{*}, F, X\right)
$$

yielding $(* *)$.

\section{Acknowledgement}

The authors thank the referees for their very valuable and detailed suggestions including giving counterexamples and shorter proofs for Theorem 2 and Theorem 3. 


\section{REFERENCE}

[1] Dunham, C. B. and ZHU, C. Z., Continuous dependence of the local strong unicity constant on domain. Aequationes Math. 46 (1993), 229-242.

Department of Mathematics,

Shanghai University of Science and Technology, Shanghai, 201800,

China.

Department of Computer Science, University of Western Ontario, London, Ontario N6A 5B7, Canada. 